\title{
TV-rate Atomic-resolution STEM Imaging
}

Ryo Ishikawa $^{1}$, Yu Jimbo ${ }^{2}$, Mitsuhisa Terao ${ }^{3}$, Masashi Nishikawa ${ }^{3}$, Yujiro Ueno ${ }^{1}$, Shigeyuki Morishita $^{2}$, Masaki Mukai $^{2}$, Naoya Shibata ${ }^{1}$ and Yuichi Ikuhara ${ }^{1}$

${ }^{1}$ University of Tokyo, Bunkyo-ku, Tokyo, Japan, ${ }^{2}$ JEOL Ltd., Akishima, Tokyo, Japan, ${ }^{3}$ JEOL, Tokyo, Tokyo, Japan

Since the development of aberration correction technology, atomic-resolution imaging is routinely available in STEM and the spatial resolution is now reached into deep sub-ångström down to 40.5 pm [1]. In addition to the electron optics, detectors such as complementary metal-oxide-semiconductor (CMOS) camera and silicon drift detector (SSD) have also significantly improved. Although static materials' analyses are remarkably progressed with the aid of these technologies, it is still lacking in the capability of dynamic observations in atomic-resolution STEM imaging. This is because the present scanning probe system takes a finite time to form an atomic-resolution image, and the temporal resolution is normally in a second per frame [2]. Therefore, in-situ observations are mostly performed in TEM mode owing to their high reading-out time of recent CMOS cameras. Towards high temporal resolution STEM imaging, several approaches have been proposed such as sparse or spiral scanning of the electron probe $[3,4]$, which can reduce the number of sampling pixels or avoid fly-back time. However, the demonstrated temporal resolutions are still in the same order or even worse, compared with the current STEM imaging. In this study, we have developed a new scanning probe system, which has electromagnetic scan coils with a considerably small inductance combined with a short lifetime scintillator for annular dark-field (ADF) STEM. Using this probe scanning system, we have achieved 25 frames per second (fps) with the image size of $512 \times 512$ pixels in atomic-resolution ADF STEM imaging, where the acquisition time is $83 \mathrm{~ns} / \mathrm{pix}$ and the fly-back time for respective line scans is $35 \mu \mathrm{s}$. The present scanning system leads to the temporal resolution of $40 \mathrm{msec}$.

Figure 1 shows the frame rate as functions of acquisition time and fly-back time for different image sizes. To achieve TV-rate observations with the image size of $512 \times 512$ pixels, the acquisition time and the flyback time should be smaller than $100 \mathrm{~ns} /$ pix and $50 \mu \mathrm{s}$, respectively. In such a short acquisition time, it may require a very high beam current and we here consider the electron dose effects on the visibility of the atomic column in ADF-STEM. We have simulated the electron dose effect on the visibility of atomic columns by adding Poisson noises in the simulated images [5]. Figure 2 shows the Poisson noise added simulated ADF-STEM images of $\mathrm{SrTiO}_{3}$ viewed along the [001] direction, where we used two parameters of pixel size (pm/pix) and electron dose per pixel (or pixel dose). Although we can obtain a large field-ofview with the larger pixel size such as $30 \mathrm{pm} / \mathrm{pixel}$, the image is strongly suffered by Poisson noise. It is therefore the pixel size should be smaller than 10 or $20 \mathrm{pm} / \mathrm{pix}$. As indicated by arrowheads, Ti-O atomic column becomes visible with the higher pixel dose of $60 \mathrm{e}^{-} / \mathrm{pix}$, corresponding to the conditions (beam current and acquisition time): (i) $192 \mathrm{pA}$ with $50 \mathrm{~ns} /$ pix or $96 \mathrm{pA}$ with $100 \mathrm{~ns} / \mathrm{pix}$. We will discuss the details of the developed scanning coil system and performance of the microscope: TV-rate acquisition of atomic-resolution ADF-STEM imaging [6]. 

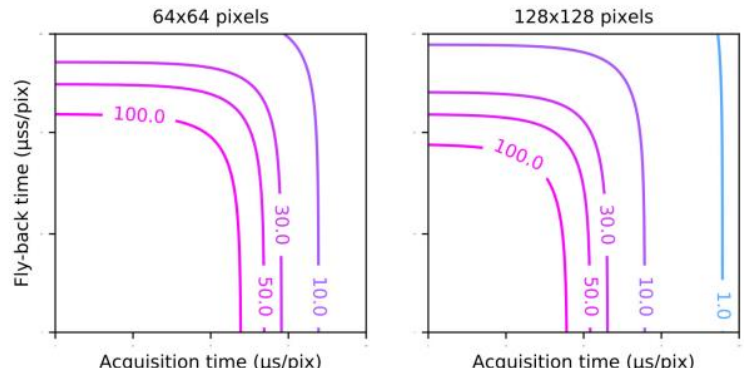

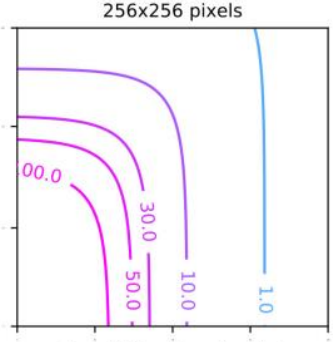

Acquisition time ( $\mu \mathrm{s} / \mathrm{pix})$

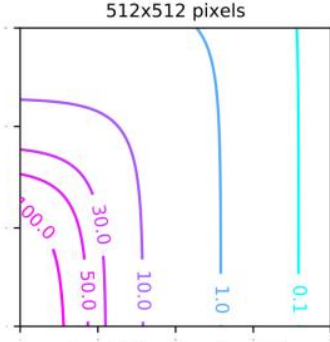

Acquisition time ( $\mu \mathrm{s} / \mathrm{pix})$

Figure 1. Frame rate (fps) as functions of acquisition time ( $\mu \mathrm{s} / \mathrm{pix})$ and fly-back time ( $\mu \mathrm{s} / \mathrm{pix})$, where the image sizes are given on the top.

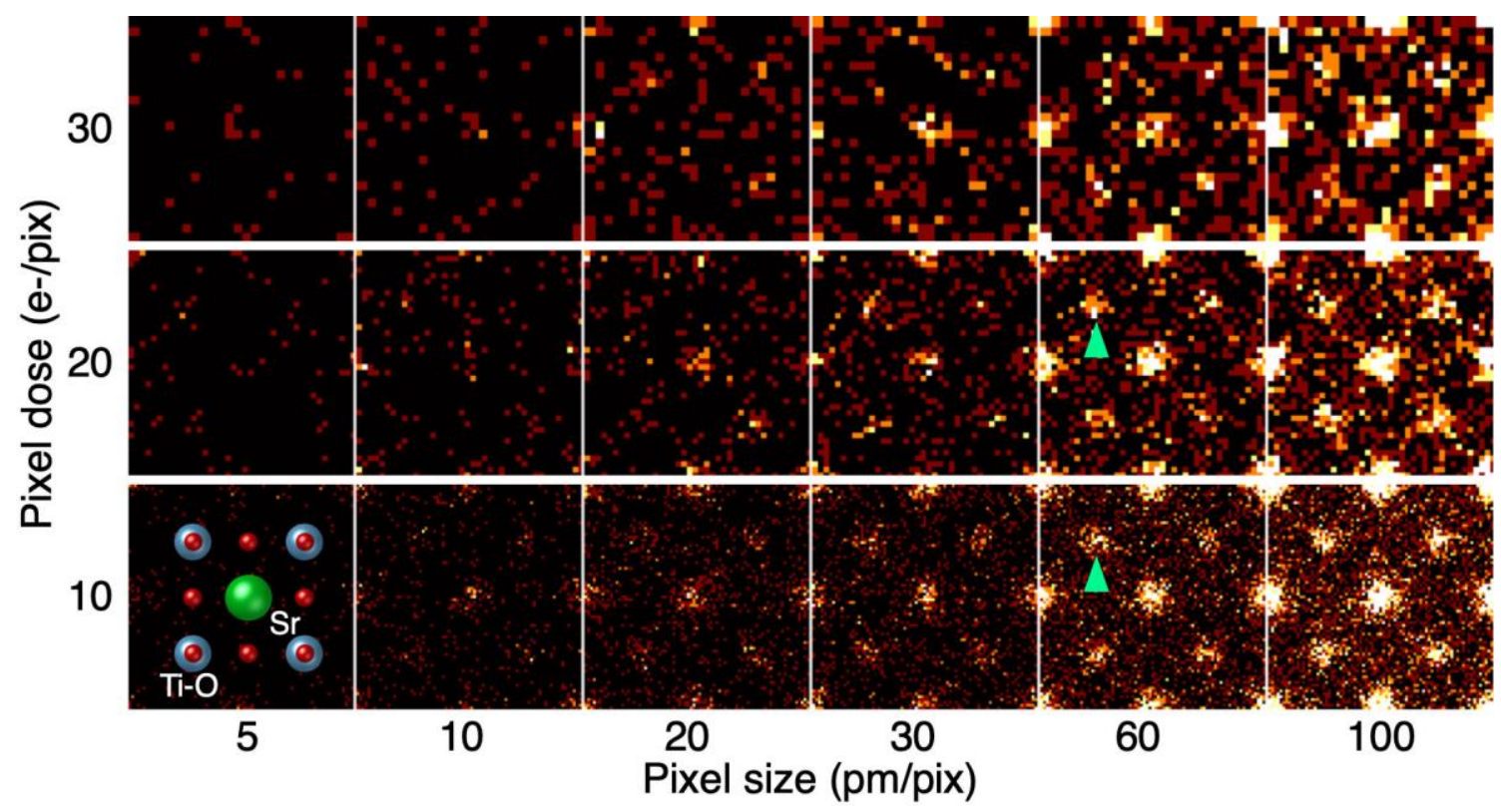

Figure 2. Poisson noise added simulated ADF-STEM images of SrTiO3 viewed along the [001] orientation.

References

[1] S. Morishita et al, Microscopy 6746 (2018).

[2] R. Ishikawa et al, Phys. Rev. Lett 113155501 (2014).

[3] A. Béché et al, Appl. Phys. Lett. 108093103 (2016).

[4] X. Sang et al, Adv. Strct. Chem. Imag. 2: 6 (2016).

[5] R. Ishikawa et al, Appl. Phys. Lett. 109163102 (2016).

[6] A part of this work was supported by Grant-in-Aid for Specially Promoted Research "Atom-by-atom imaging of ion dynamics in nano-structures for materials innovation" (Grant No. JP17H06094) from JSPS. 